\title{
Implementation of a Hepatitis C Treatment Program Into a Primary Care Residency Clinic
}

Morgan Adams Rhodes, PharmD, BCACP | Mark Humphrey, MD, MPH

PRiMER. 2021;5:1.

Published: 1/22/2021 | DOI: 10.22454/PRiMER.2021.532368

\section{Abstract}

Background and Objectives: Hepatitis $\mathrm{C}(\mathrm{HCV})$ is a commonly diagnosed disease state in primary care. Regimen simplification has made eradication possible with improvements in treatment and improved access through primary care physician (PCP) education. Little has been published discussing the role of resident physicians in the treatment of HCV. We implemented an HCV treatment program to increase access to HCV care, identify effective training methods, and examine the efficacy of resident physician treatment. Objectives were to increase the number of patients treated, improve resident confidence in ability to treat $\mathrm{HCV}$, and increase the likelihood that they will continue to treat patients after graduation.

Methods: A curriculum to train physicians to treat HCV was developed and implemented in a large family medicine residency program. This was a single-center implementation, with a retrospective chart review of patient data and anonymous survey of clinicians for curriculum assessment. We analyzed data using descriptive statistics.

Results: The resident physician survey had a $92.3 \%$ response rate $(n=36)$. Precurriculum, $94.4 \%$ of residents were not confident in their ability to treat HCV. After program implementation, $25 \%$ of residents were confident in treating HCV. The most effective educational interventions involved the multidisciplinary team. To date, 30 patients have started treatment since 2017. Of the patients who completed treatment, 23 patients achieved a virologic cure.

Conclusion: Treating patients for HCV at a residency clinic increased physician confidence in evaluating and addressing this chronic disease and resulted in a cure of all patients treated.

\section{Introduction}

Hepatitis $\mathrm{C}(\mathrm{HCV})$ is a commonly diagnosed disease with an estimated 3.5 million Americans currently living with chronic HCV. ${ }^{1}$ Current medications for HCV are well tolerated, simple, oral regimens with cure rates over $93 \% .^{2,3}$

To encourage broad treatment availability, the American Academy of Family Physicians, in conjunction with specialists, have argued that primary care physicians (PCPs), with training, should have unrestricted access to prescribing medications for HCV, as trained PCPs have demonstrated efficacy in treating HCV. ${ }^{4,5}$ Models for education include the Extension for Community Healthcare Outcomes (ECHO) model (telehealth specialist 
consultation) and didactic training. In both models, PCPs have achieved similar sustained virologic response $\left(\mathrm{SVR}_{12}\right)$ rates as specialists. ${ }^{6,7}$

Multidisciplinary teams, including pharmacists, are financially feasible and can reduce physician inertia by assisting with tasks such as insurance approval and medication monitoring to help PCPs achieve high SVR 12 rates. $^{8-10}$

With the evidence available to support PCPs treating HCV, these interventions need to be brought to residency programs, where little research has been done to date. ${ }^{11}$ Our objectives were to increase the number of patients treated, increase resident confidence in their ability to treat, increase the likelihood that residents will continue to treat patients after graduation, and demonstrate the efficacy of residents in treating HCV.

\section{Methods}

\section{Training Program}

The residency practice is a low-income, urban, hospital-based clinic that cares for approximately 10,000 patients. To address training gaps, we initiated an HCV treatment program in June 2017 for residents and faculty (Table 1). This included educational interventions with previously documented successes. ${ }^{6-10}$ Because PCPs in South Carolina are required to have specialist consultation to prescribe HCV medications for some insurances, our program included instruction in the use of telehealth.

\section{Evaluation Methods}

Evaluation consisted of an anonymous survey of residents and a retrospective chart review (Appendix A). To assess the educational interventions, we developed an anonymous pre/postsurvey to assess resident confidence in treating HCV for residents graduating from 2018 to 2021. We asked respondents the number of patients they had treated, the most effective educational intervention, and their intention or current practice of treating patients after graduation. Respondents were also asked to rate their confidence in treating HCV preand postcurriculum, and their confidence in the multiple components of HCV management on a Likert scale. We analyzed survey responses by descriptive statistics.

We collected data on patient characteristics via retrospective chart audit. We collected patient demographics and the level of training for the physician treating each patient. Cure was defined as an undetectable viral load 12 weeks after completion of treatment $\left(\mathrm{SVR}_{12}\right)$. We analyzed results using descriptive statistics.

The Institutional Review Board at Prisma Health-Midlands reviewed and approved this study.

\section{Results}

The survey was sent to 30 residents and nine program graduates. A total of 36 residents or graduates completed the survey (92.3\% response rate). Among the residents surveyed, 21 (58.3\%) had treated at least one patient for HCV. Of the seven graduates who answered the survey, two were currently treating patients for $\mathrm{HCV}$. Of the 29 current residents, 17 (58.6\%) who had treated one or more patients for HCV, 16 intend to treat patients for HCV after graduation, with 12 unsure.

Precurriculum, 34 (94.4\%) residents were not confident at treating HCV. After the curriculum implementation, nine $(25 \%)$ residents are confident in treating HCV with only six residents (16.7\%) still not confident (Figure 1). Residents who treated at least two patients felt somewhat confident or confident after the program. Residents felt most confident in ordering pretreatment labs and screenings and selecting vaccines. Residents felt least confident in selecting medications and managing drug interactions and side effects. The majority of residents 
(80\%) cited the multidisciplinary team as the most effective educational intervention (Table 2).

A total of 30 patients have started or completed treatment. Patients were aged an average of 58.4 years (range 29-76 years, Table 3). Of the patients treated, $76.7 \%$ were treated by a resident physician, mainly PGY2 and PGY3s (70\%).

A majority of patients (20) achieved an undetectable viral load at week 4 of treatment. Of the patients who completed treatment, an undetectable viral load $\left(S R_{12}\right)$ was achieved by all 23 patients ( $100 \%$ cure rate), with seven patients yet to have their $\mathrm{SVR}_{12}$ completed.

\section{Discussion}

This curriculum increased capacity to provide broad-spectrum primary care to our patients to include HCV care. The majority of patients diagnosed with HCV in our clinic are currently treated by their PCP instead of specialists.

A curriculum to train family medicine residents to treat HCV was effective in improving resident confidence in treating HCV and successful in curing the disease. To our knowledge, this is the first study that demonstrates the efficacy of primary care trainees in treating HCV. In a recent survey of family medicine residency program directors, most (61.9\%) believe their programs are integral in building the future workforce to treat HCV. ${ }^{12}$ Similar curricula could be implemented across family medicine residency programs to increase access to care during residency and once in practice.

We utilized a combination of educational interventions that could be applied broadly in residency programs. Resident confidence appeared to be related to the number of patients treated during residency, with residents treating at least two patients identifying as somewhat confident or confident in treating HCV. After the curriculum initiation, all but one current resident planned to treat HCV in the future or were unsure. We believe this is a positive step in developing PCPs' capacity and willingness to treat HCV. Additional research is needed to understand why residents decide to treat or not to treat HCV upon graduation.

This study has several limitations, including the small study size of residents and patients treated, and lack of HIV coinfected patients. As a result, we were unable to power the study to measure statistical differences in confidence and likelihood of treating HCV after graduation. Although the survey was anonymous, the survey was disseminated by study investigators, leading to potential response bias. Additionally, for the patient outcomes, almost one-quarter of the patients were treated by attending physicians. Although the attending physicians were not the target subjects for the intervention, the physicians followed a similar process and had similar outcomes to the residents' patients. Finally, only $25 \%$ of residents felt confident in treating HCV after the curriculum. Since number of patients treated seemed to be related to confidence, we believe that continuing this curriculum and increasing resident opportunities to treat patients will help to address this limitation. A quality improvement project is currently underway to improve HCV screening for all patients aged 18-79 years according to the recently updated United States Preventive Services Task Force Recommendations. ${ }^{13}$

The curriculum has continued and will focus on case-based scenarios for medication management. Scenarios will specifically address areas of need such as drug interactions and treatment regimen selection. Improved screening may create additional opportunities for diagnosis and treatment of HCV at our clinic. A multifaceted HCV curriculum allowed residents to feel more confident in treating HCV and resulted in effective cure of all patients treated at the clinic. 


\section{Tables and Figures}

Table 1: HCV Curriculum

\begin{tabular}{|c|c|c|}
\hline Activity & Frequency & Description \\
\hline $\begin{array}{l}\text { Didactics and topic } \\
\text { discussions }\end{array}$ & Every $12-18$ months & $\begin{array}{l}\text { Formal, hour-long lectures are integrated into the resident didactic } \\
\text { curriculum cover the following topics: screening, pretreatment monitoring/ } \\
\text { screening, vaccinations, treatment selection, during and after treatment } \\
\text { monitoring, and medication access. Lectures are recorded. Residents also } \\
\text { participate in } 1: 1 \text { topic discussions with faculty pharmacist about HCV. }\end{array}$ \\
\hline $\mathrm{HCV}$ telehealth initiative & $\begin{array}{l}\text { First, third, and fourth } \\
\text { Wednesdays of every } \\
\text { month }\end{array}$ & $\begin{array}{l}\text { Hosted by the South Carolina AIDS Education and Training Center } \\
\text { (AETC), primary care physicians submit and present cases to be evaluated } \\
\text { and discussed with infectious disease and hepatology specialists. } \\
\text { Recommendations for care are provided. Resident and attending } \\
\text { physicians were encouraged to submit complicated cases, and all } \\
\text { cases that required specialist consultation (ie, Medicaid, Medicare) were } \\
\text { submitted (https://www.seaetc.com/programs-services/viral-hepatitis/) }\end{array}$ \\
\hline Multidisciplinary care team & As needed & $\begin{array}{l}\text { After diagnosis of chronic hepatitis } C \text { is made, physicians contact } \\
\text { pharmacy team (pharmacist faculty, pharmacy resident, pharmacy student), } \\
\text { mainly via electronic health record messaging. Patient is reviewed by a } \\
\text { pharmacy team member and treatment plan and monitoring is discussed } \\
\text { with primary care physician. Pharmacy team assist with task such as } \\
\text { prior authorizations, medication access, patient follow-up, and patient } \\
\text { counseling. }\end{array}$ \\
\hline
\end{tabular}

Table 2: Most Effective Educational and Practice Modification Strategies for Teaching Management of Hepatitis C: Results of Resident Survey $(\mathrm{N}=36)$

\begin{tabular}{|l|c|c|}
\hline \multicolumn{1}{|c|}{ Intervention } & $\begin{array}{c}\text { Was This } \\
\text { Intervention } \\
\text { Effective? } \\
\text { Yes Responses (\%) }\end{array}$ & $\begin{array}{c}\text { Was This the Most } \\
\text { Effective Intervention? } \\
\text { Yes Responses (\%) }\end{array}$ \\
\hline Pharmacist embedded in practice & 86 & 61 \\
\hline Formal presentations (lecture) & 83 & 11 \\
\hline Pharmacy-based case management & 75 & 19 \\
\hline Worksheet on HCV management & 36 & 6 \\
\hline Hepatitis C teleconference & 33 & 6 \\
\hline
\end{tabular}


Figure 1: Confidence in Treating Hepatitis C

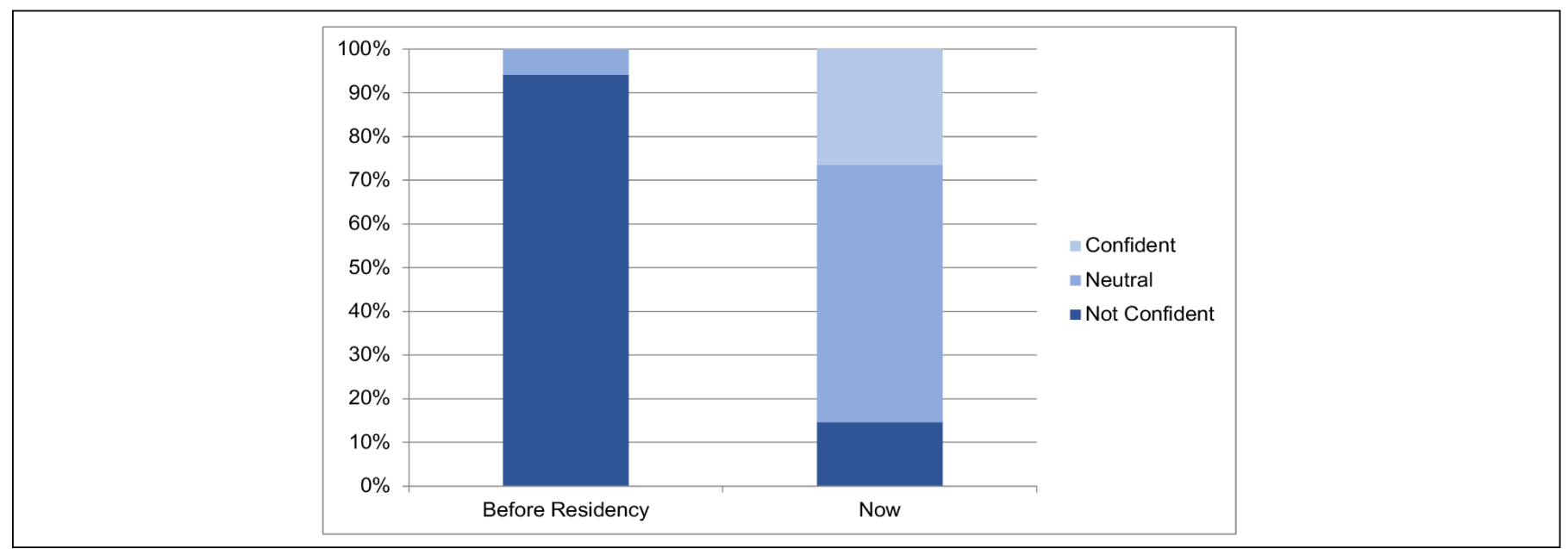

Figure 2: Confidence in Components of HCV Treatment $(n=36)$

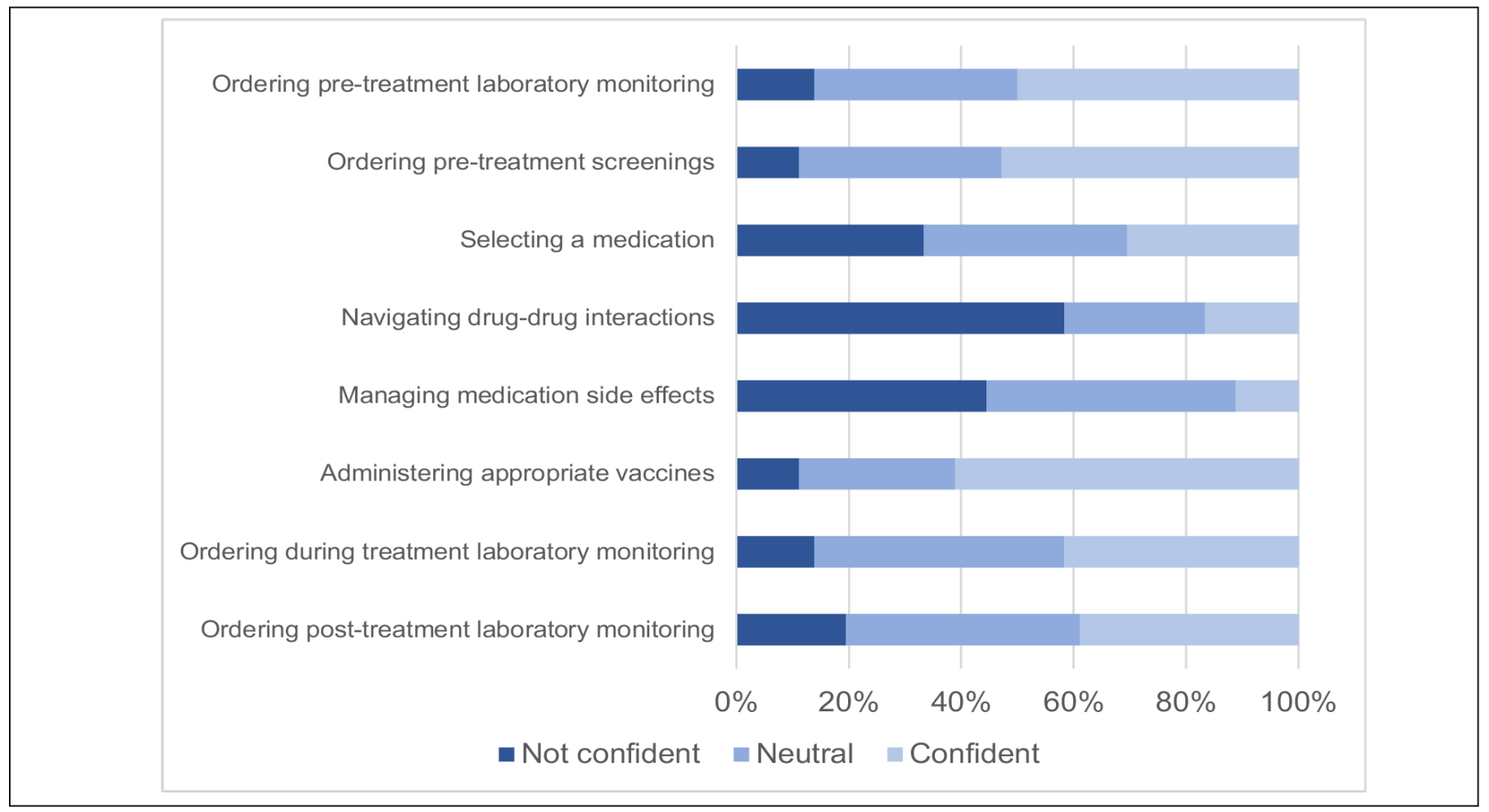


Table 3: Patient Characteristics ( $\mathbf{N}=\mathbf{3 0})$

\begin{tabular}{|l|l|}
\hline \multicolumn{1}{|c|}{ Characteristics } & \multicolumn{1}{c|}{ Number of Patients (\%) } \\
\hline Genotype & $21(70)$ \\
$1 \mathrm{a}$ & $6(20)$ \\
$1 \mathrm{~b}$ & $1(3.33)$ \\
2 & $1(3.33)$ \\
3 & $1(3.33)$ \\
Unknown & $15(50)$ \\
\hline Cirrhosis (Metavir F3 or F4) & $16(53.3)$ \\
\hline Female & \\
\hline Insurance Type & $3(10)$ \\
Uninsured & $6(20)$ \\
Medicaid & $11(36.7)$ \\
Medicare & $10(33.3)$ \\
\hline Commercial & \\
Comorbid Chronic Disease & $9(30)$ \\
Diabetes & $24(80)$ \\
Hypertension & $1(3.33)$ \\
\hline Chronic kidney disease & \\
Social History & $5(16.7)$ \\
History of alcohol use disorder & $9(30)$ \\
\hline History of substance use disorder & \\
\hline Level of Training of Treating Physician & $2(6.7)$ \\
\hline PGY1 & $10(33.3)$ \\
PGY2 & $11(36.7)$ \\
PGY3 & $7(23.3)$ \\
Attending & \\
\hline Medication & $12(40)$ \\
Glecaprevir/pibrentasvir 100 mg/40 mg & $9(30)$ \\
Sofosbuvir/velpatasvir $400 \mathrm{mg} / 100 \mathrm{mg}$ & $9(30)$ \\
\hline Ledipasvir/sofosbuvir 90 mg/400 mg & \\
\hline
\end{tabular}

\section{Acknowledgments}

The authors thank University of South Carolina School of Pharmacy students Alyssa Bryan and Julia Brown, and University of South Carolina School of Medicine students Tushar Patel and Matthew Lancaster for assistance in chart audits. The authors also thank the current and former residents of the Prisma HealthMidlands/University of South Carolina Family Medicine Residency Program for their participation in the survey.

\section{Corresponding Author}

Morgan Adams Rhodes, PharmD, BCACP

3209 Colonial Drive, Columbia, SC 29203. 803-434-2072. Fax: 803-434-7529

morgan.rhodes@uscmed.sc.edu

\section{Author Affiliations}

Morgan Adams Rhodes, PharmD, BCACP - University of South Carolina School of Medicine, Department of Family and Preventive Medicine

Mark Humphrey, MD, MPH - University of South Carolina School of Medicine, Department of Family and Preventive Medicine

\section{References}


1. Centers for Disease Control and Prevention. Hepatitis Statistics. https://www.cdc.gov/hepatitis /statistics/DiseaseBurden.htm. Accessed June 25, 2019.

2. American Association for the Study of Liver Diseases. Initial Treatment of Adults with HCV Infection. https://www.hcvguidelines.org/treatment-naive Updated August 27, 2020. Accessed June 25, 2019.

3. Hepatitis C Online. Cost and Access to Direct-Acting Antiviral Agents. https://www.hepatitisc.uw.edu /pdf/evaluation-treatment/cost-access-medications/core-concept/all. Accessed June 25, 2019.

4. Simoncini GM, Koren DE. Hepatitis $\mathrm{C}$ update and expanding the role of primary care. J Am Board Fam Med. 2019;32(3):428-430. doi:10.3122/jabfm.2019.03.180286

5. American Academy of Family Physicians. Academy Lauds Bill That Would Relieve Some PPE Expenses. https://www.aafp.org/news/health-of-the-public/20160420hepcaccess.html Accessed June 11, 2019.

6. Arora S, Thornton K, Murata G, et al. Outcomes of treatment for hepatitis C virus infection by primary care providers. N Engl J Med. 2011;364(23):2199-2207. doi:10.1056/NEJMoa1009370

7. Kattakuzhy S, Gross C, Emmanuel B, et al; and the ASCEND Providers. Expansion of treatment for hepatitis $C$ virus infection by task shifting to community-based nonspecialist providers: a nonrandomized clinical trial. Ann Intern Med. 2017;167(5):311-318. doi:10.7326/M17-0118

8. Lasser KE, Heinz A, Battisti L, et al. Budgetary impact analysis of a primary care-based hepatitis C treatment program: effects of 340B drug pricing program. Ann Fam Med. 2017;15(3):258-261. doi:10.1370/afm.2069

9. Jones EA, Linas BP, Truong V, Burgess JF, Lasser KE. Budgetary impact analysis of a primary care-based hepatitis C treatment program: Effects of 340B Drug Pricing Program. PLoS One. 2019;14(3):e0213745. doi:10.1371/journal.pone.0213745

10. Couri T, Gupta T, Weyer G, Aronsohn A. Pay it forward: building capacity to treat hepatitis $C$ by training our own residents. Hepatology. 2018;68(5):2004-2007. doi:10.1002/hep.30101

11. Facente SN, Burk K, Eagen K, Mara ES, Smith AA, Lynch CS. New treatments have changed the game: hepatitis $C$ treatment in primary care. Infect Dis Clin North Am. 2018;32(2):313-322. doi:10.1016/j.idc.2018.02.012

12. Webb Camminati C, Simha A, Kolb NR, Prasad R. Intent to build hepatitis $C$ treatment capacity within family medicine residencies: a nationwide survey of program directors: a CERA study. Fam Med. 2016;48(8):631-634.

13. US Preventive Services Task Force. Hepatitis C Virus Infection in Adolescents and Adults: Screening. https://www.uspreventiveservicestaskforce.org/uspstf/recommendation/hepatitisc-screening\#fullrecommendationstart. Published March 2, 2020. Accessed October 10, 2020. 